\title{
Current Data on the Profile of Patients Hospitalized for Stroke in a Neurology Department in West Africa: Abidjan (Ivory Coast)
}

\author{
Aka-Anghui Diarra Evelyne ${ }^{1}$, Zakaria Mamadou ${ }^{1}$, Mourtada Wardatou Dine ${ }^{2}$, \\ Kouassi Kouamé Léonard ${ }^{3}$, Yapo Ehounoud C. ${ }^{1}$ \\ ${ }^{1}$ Faculty of Medical Sciences, Department of Neurology, University Hospital of Cocody, Félix Houphouët Boigny University, Abidjan, Ivory \\ Coast \\ ${ }^{2}$ Faculty of Medical Sciences, Department of Infectious and Tropical Diseases, University Hospital of Treichville, Félix Houphouët Boigny \\ University, Abidjan, Ivory Coast \\ ${ }^{3}$ Faculty of Medical Sciences, Department of Neurology, University Hospital of Yopougon, Félix Houphouët Boigny University, Abidjan, \\ Ivory Coast
}

\section{Email address:}

evediarra@yahoo.fr (Aka-Anghui D. Evelyne), mamadouzakaria9@gmail.com (Z. Mamadou),warda.dine@gmail.com (M. Wardatou Dine), lkouassi93@yahoo.fr (K. K. Léonard), constanceyapoehounoud@gmail.com (Yapo E. C.)

\section{To cite this article:}

Aka-Anghui Diarra Evelyne, Zakaria Mamadou, Mourtada Wardatou Dine, Kouassi Kouamé Léonard, Yapo Ehounoud C.. Current Data on the Profile of Patients Hospitalized for Stroke in a Neurology Department in West Africa: Abidjan (Ivory Coast). American Journal of Psychiatry and Neuroscience. Special Issue: Clinical Neurosciences in Tropical Practice. Vol. 3, No. 5-1, 2015, pp. 14-21. doi: 10.11648/j.ajpn.s.2015030501.14

\begin{abstract}
Background and purpose: With a hospital prevalence oscillating between $28 \%$ and $32 \%$ the strokes represent the first cause of hospitalization in our department [1] Stroke is emerging as a leading cause of preventable death and disability in adults in many developing nations. The general objective was to describe the profile of patients. Problem: The management of stroke is real public challenges under tropic regions. Methodology: We prospectively studied consecutive patients presenting to the neurology unit of Abidjan- Cocody, over a one year period (March 2012-February 2013). The socio-demographic, clinical data, the risk factors as well as the CT scan findings were collected in the patients registers. Results: The hospital prevalence of stroke was $28.3 \%$. The average age was 58 years, and a quarter of patients had less than 50 years. A slight male predominance was noted with a sex ratio of 1.13 . The average admission time was 6.7 days and the average period of hospitalization was 10 days. The main risk factor listed was hypertension (79.3\%). The percentage of recurrent stroke was $21.6 \%$. The main reasons for admission were: a motor deficit (100\%) and headache (88.4\%). Computed tomography has allowed the diagnosis of $59 \%$ of ischemic stroke especially the MCA territory and $41 \%$ of hemorrhagic strokes, mostly basal ganglia. The main causes were, for ischemic stroke, $47 \%$ of vascular causes, and hemorrhagic stroke, $74 \%$ of micro aneurysms associated with hypertension. Exploration difficulties were responsible for a significant percentage of undetermined causes. We recorded $24.1 \%$ of deaths. Conclusion: As in lot of developing African countries, the study suffers from strokes undetermined causes due to lack of financial means, and the failure of the technical platform.
\end{abstract}

Keywords: Stroke, CT, Cardiovascular Risk Factors, Etiologies, Death

\section{Introduction}

According to the World Health Organization, stroke is defined as the rapid development of localized or global signs of cerebral dysfunction with symptoms lasting more than 24 hours, which can lead to death without any other apparent cause apart from a vascular origin.
In our practice, cerebrovascular diseases are the most common acute neurological disorders and the first neurological cause of hospitalization. In Ivory Coast, strokes are at the forefront of neurological disorders. They represent more than $32 \%$ of the hospitalizations in the Neurology 
department. If the mortality rate is down from $43.9 \%$ [1] to $24.1 \%$, it remains a concern for the clinician.

\section{Methodology}

Prospective descriptive study over a period of one year from March 1st, 2012 to February 28th, 2013; performed in the largest neurology department of the capital which counts 02, located in the University Hospital of Cocody. It has a 32 bed capacity and lacked of stroke unit at the time of the study. Inclusion criteria were: all patients admitted during the study period for stroke confirmed by brain CT scan. Thus every patient with a neurological deficit of non traumatic sudden onset with CT confirmation lesions was included. The hypertension was defined by blood pressure $\geq 140$ / $90 \mathrm{~mm}$ $\mathrm{Hg}$. The scanner was realized with a monobarette $\mathrm{X}$ device, axial, sagittal, and coronal slides of floors above and under tentorial without and with contrast injection products (gadolinium).

The cerebral venous thrombosis, the absence of CT, all focal motors deficits with non-imaging for stroke was exclusion criteria. For ethical reasons, we requested the consent of patients or families, and the sheets were indicated Privacy object.

The following parameters were studied:

- Socio-demographic data and risk factors

- The admission period and performing a brain imaging.

- The TOAST criteria were used for the classification of etiologies of ischemic stroke.

- The complications during hospitalization and stroke mortality.

For statistical analysis, we used the chi-square test with a probability value of 0.05 set as the statistical significance level.

\section{Results}

\subsection{Epidemiological Characteristics}

During the study period, 819 patients were hospitalized including 232 cases of stroke, with a hospital prevalence of $28.3 \%$. Patient age ranged between 20 and 94 years with an average of 58 years. The under 50 accounted for one quarter of the workforce (25\%) (TABLE I). The sex ratio $\mathrm{M} / \mathrm{F}$ was 1.13. Stroke significantly affected more women than men after age $70[\mathrm{khi} 2(\chi 2=12.094 \mathrm{P}=0.0005)]$.

Table I. Distribution of patients according to age.

\begin{tabular}{lll}
\hline Ages & Workforce & Percentages(\%) \\
\hline $20-29$ & 06 & 02,6 \\
$30-39$ & 15 & 06,5 \\
$40-49$ & 36 & 15,5 \\
$50-59$ & 49 & 21,1 \\
$60-69$ & 73 & 31,5 \\
$70-79$ & 36 & 15,5 \\
$80-94$ & 17 & 07,3 \\
TOTAL & 232 & 100 \\
\hline
\end{tabular}

The study population was predominantly made up of people with no income (59.9\%), no fixed employment, pensioners, housewives and unemployed.

\subsection{Clinical Features}

The average admission time was 6.17 days, less than $20 \%$ of patients admitted within 24 hours and $50 \%$ in the 72 hours following stroke. The average hospital stay was 10.43 days with a range of 1-94 days.

At the examination, hypertension was the major vascular risk factor found as family history in $44 \%$ of cases and as a personal history with nearly $80 \%$ of our patients. A stroke recurrence was found in $21 \%$ of cases (TABLE II).

The study population consisted of right-handed in $92 \%$ of cases and $13 \%$ of non-Ivorian patients $(87 \%$ of Ivorian nationality). The assessment of vigilance on admission found $52.2 \%$ of patients with a Glasgow score less than 11 (Table III). The main reasons for admission were: motor deficit $(100 \%)$, headache $(88.4 \%)$, vigilance disorders $(52.1 \%)$, vomiting (45,7\%) and seizures (24,6\%) (Figure 1).

The motor deficit was hemiplegia in $59 \%$ of cases, on the left side $(50.6 \%)$, most often proportional $(80 \%)$. The clinical examination revealed a pyramidal syndrome in $100 \%$ of cases, a deficit of higher cerebral functions in $65.9 \%$ dominated by Broca's aphasia (35.3\%) and a sensory disorder in $44.5 \%$ of cases (Figure 2 ).

We compared the value of meningea syndrome depending on the type of stroke: it was significantly higher in ICH (Table IV).

Table II. Distribution by risk factors.

\begin{tabular}{ll}
\hline \multirow{2}{*}{ Risk factors } & Total $(\%)$ \\
\cline { 2 - 2 } & Number $(\%)$ \\
\hline hypertension & $184(79,3)$ \\
diabetes mellitus & $42(18,1)$ \\
alcohol & $63(27,2)$ \\
tobacco smoking & $49(21,1)$ \\
heart disease & $15(6,5)$ \\
stroke & $50(21,6)$ \\
dyslipidemia & $2(0,9)$ \\
oral contraceptives & $10(4,3)$ \\
anticoagulant therapy & $2(0,9)$ \\
\hline
\end{tabular}

Table III. Distribution by the Glasgow Coma Scale (GCS) at admission.

\begin{tabular}{lll}
\hline State of consciousness (GCS) & Workforce & Percentages \\
\hline Normal =15 & 111 & 47,8 \\
Confusion (11- 14) & 50 & 21,6 \\
Coma (8 -10) & 46 & 19,8 \\
Deep coma (3-7) & 25 & 10,8 \\
Total & 232 & 100 \\
\hline
\end{tabular}

Table IV. Comparing the frequency of meningeal syndrome depending on the type of stroke.

\begin{tabular}{lllll}
\hline Type of & \multicolumn{3}{l}{ Meningeal syndrome } & Chi-square \\
\cline { 2 - 5 } stroke & Found & Not found & probability & significance \\
\hline AIS & $13(9,4 \%)$ & $125(90,6)$ & 0,0003 & S \\
ICH & $26(27,7 \%)$ & $68(72,3 \%)$ & & \\
\hline
\end{tabular}




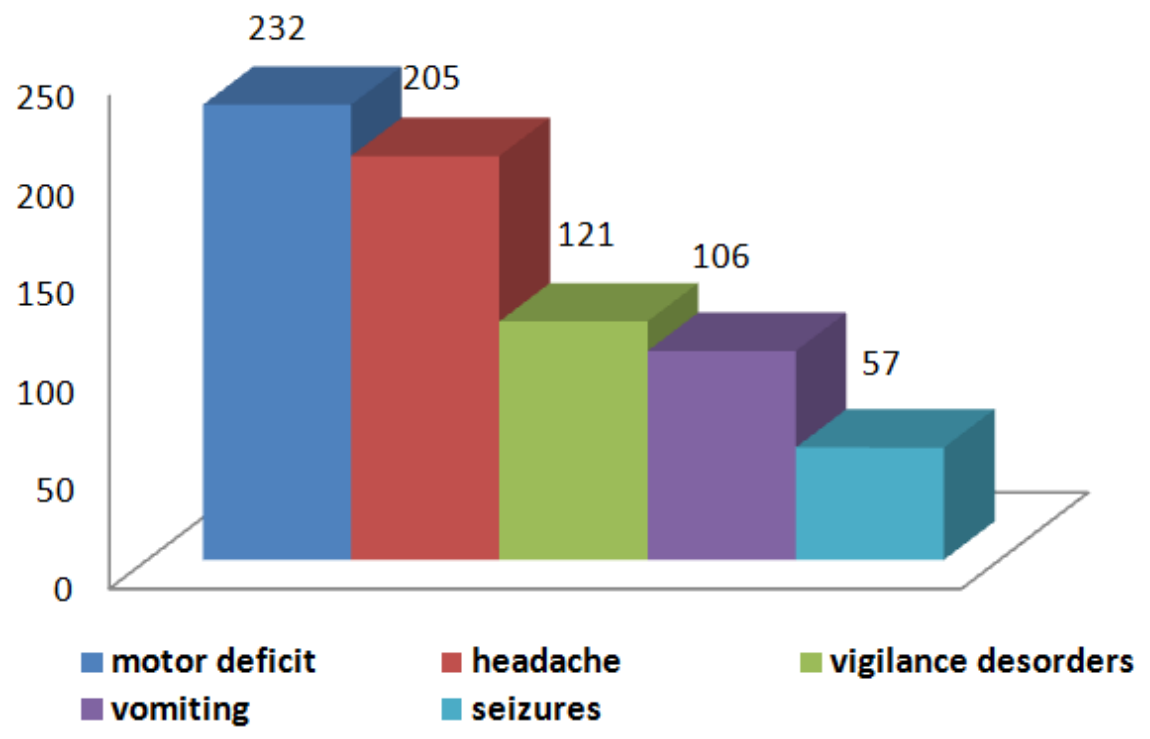

Figure 1. Distribution by early symptoms.

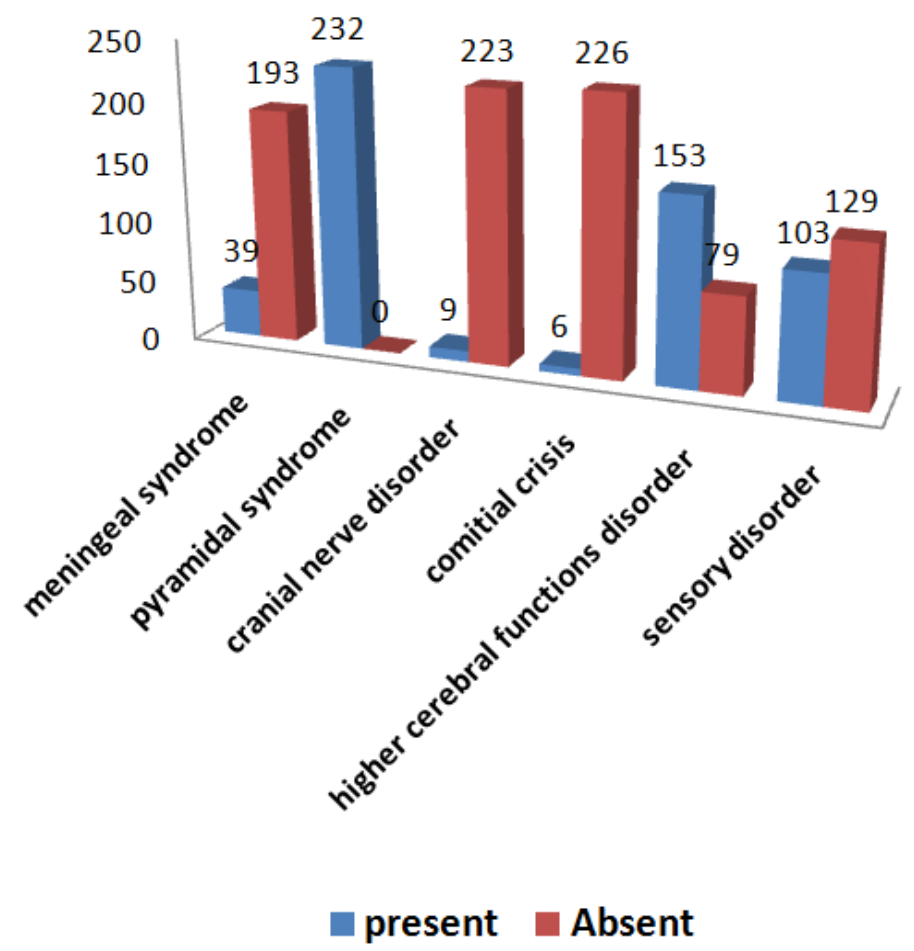

Figure 2. Distribution by syndromes and signs found on clinical examination.

\subsection{Paraclinical Features}

Brain computed tomography (CT) scan was performed in one quarter $(25.1 \%)$ of patients in the first 48 hours post stroke, with a range of 0-60 days (average completion time $=$ 4.6 days post stroke) (Table V). The mechanism of stroke was ischemia in $59 \%$ of cases, hemorrhage in $41 \%$ of cases (Figure 3). The topography of ischemic strokes interested the territory of the middle cerebral artery in $45.2 \%$ of cases and location of hemorrhagic stroke involved the basal ganglia in $60.4 \%$. The main signs associated with CT were: mass effect with $(52.5 \%)$, cerebral atrophy $(20 \%)$ and old stroke sequelae (8.6\%). CT angiography (CTA) was performed for $30 \%$ of patients with AIS; and MRI for less than $1 \%$ of cases.

Table VI reviews the major biological analyzes of patients in this study.

The main cardiovascular examination performed was ECG (44.8\%). Figure 4 indicates cardiovascular explorations made and their percentage.

The main anomaly found is left ventricular hypertrophy in $44 \%$ of $44.8 \%$ of subjects who received a cardiac evaluation.

The atherosclerotic vascular causes were implicated in $47 \%$ 
of ischemic stroke and hypertension in 74\% of hemorrhagic strokes (Figure 5- Figure 6).



Figure 3. Distribution by type of stroke on CT.

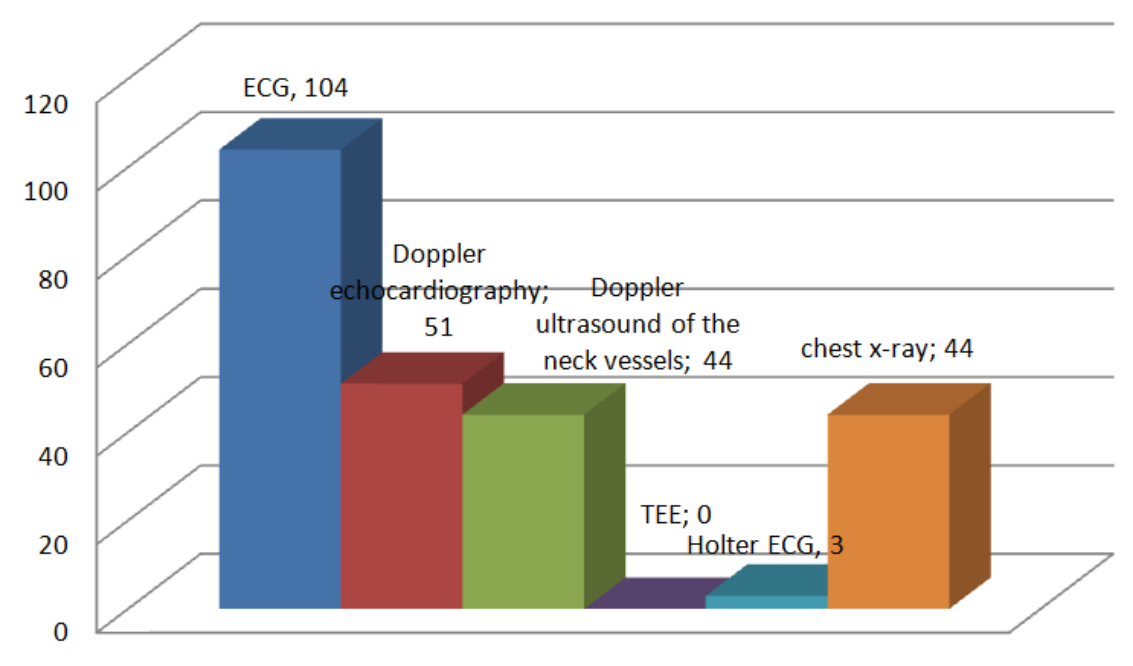

Figure 4. Distribution of the differents cardiovascular imaging.

ECG: electrocardiogram (ECG)

TEE: Transesophageal echocardiography

Holter ECG: holter electrocardiogram

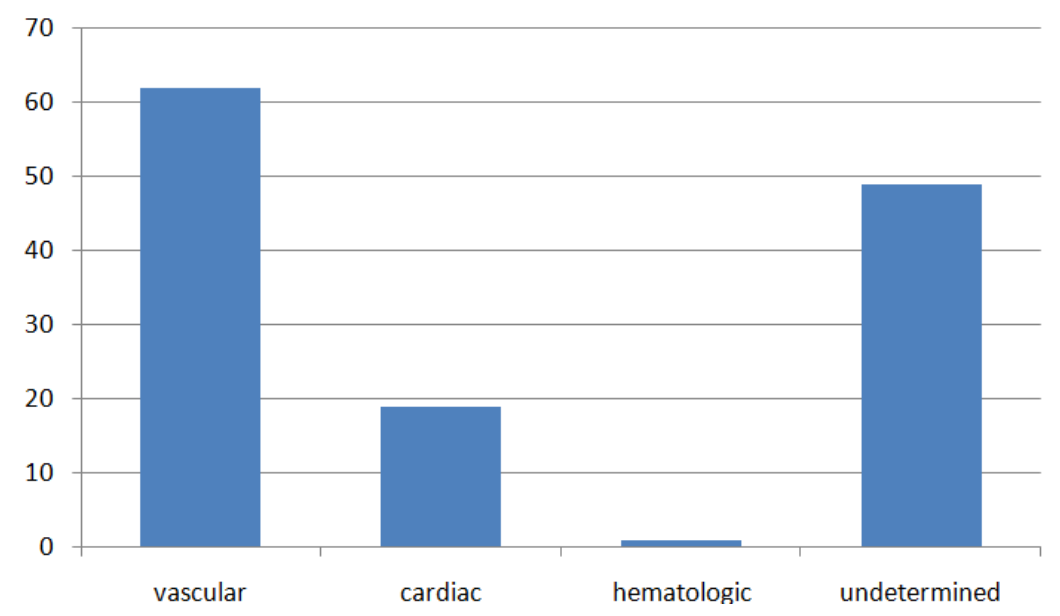

AIS $=$ Acute Ischemic Stroke

Figure 5. Distribution of patients with AIS according to etiological diagnosis ( $N=138$ number of patients diagnosed AIS). 




$\mathrm{ICH}=$ IntraCerebral Hemorrhage

Figure 6. Distribution of patients with ICH according to the etiological diagnosis.

Table $\boldsymbol{V}$. Distribution by the completion time of CT relative to early signs.

\begin{tabular}{lll}
\hline Completion time (days) & Workforce & Percentages \\
\hline$<48 \mathrm{~h}$ & 58 & 25,1 \\
3 à 5 & 107 & 46 \\
6 à 10 & 42 & 18,2 \\
11 à 20 & 12 & 6,9 \\
21 à 60 & 9 & 3,87 \\
TOTAL & 232 & 100,0 \\
\hline
\end{tabular}

Table VI. Main paraclinical tests realized.

\begin{tabular}{lll}
\hline Paraclinical tests & Workforce & Percentage \\
\hline Blood glucose & 232 & 100 \\
Complete blood count & 231 & 99,6 \\
Renal function tests & 231 & 99,6 \\
Haemostatic tests & 230 & 99,1 \\
Serum electrolytes & 230 & 99,1 \\
\hline
\end{tabular}

\begin{tabular}{lll}
\hline Paraclinical tests & Workforce & Percentage \\
\hline Liver function tests & 228 & 98,2 \\
Serum lipid profile & 206 & 88,8 \\
Lumbar puncture & 21 & 9,1 \\
\hline
\end{tabular}

\subsection{Treatment and Evolution}

The correction of cardiovascular risk factors required the prescription of antihypertensive drugs (90.5\%), statins $(51.7 \%)$ and diabetes mellitus medical care (16.4\%). The most frequently prescribed symptomatic and causal treatment is summarized in Table VII and VIII.

Evolution in hospital was marked by $37 \%$ of decubitus complications (figure 7) and a mortality rate of $24.1 \%$.

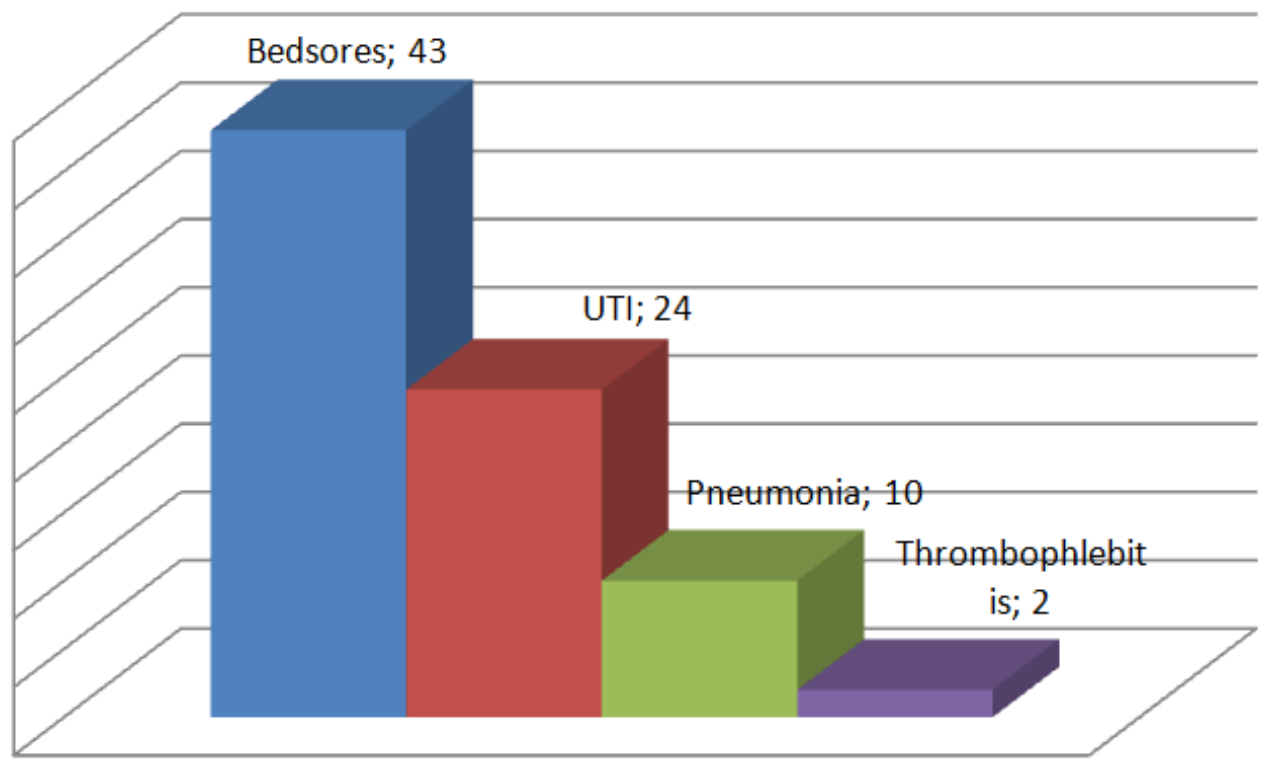

Figure 7. Types of complications during hospitalization. 
Table VII. Distribution by type of treatment.

\begin{tabular}{lll}
\hline Symptomatic treatment & Number observed & Percentages \% \\
\hline analgesics & 230 & 99,1 \\
platelet antiaggregation & 138 & 59,5 \\
agents & 26 & 11,2 \\
anticoagulation therapy & 25 & 10,8 \\
Antiepileptic drugs & 8 & 3,4 \\
cerebral oxygenator & 7 & 3,0 \\
anti-oedematous drugs &
\end{tabular}

Table VIII. Distribution by type of anti hypertensive agents.

\begin{tabular}{lll}
\hline Type of anti hypertensive agents & Workforce & Percentages \\
\hline ACEIs + diuretics combination & 142 & 67,2 \\
ACEIs & 37 & 15,9 \\
Calcium antagonists & 27 & 11,6 \\
Beta blockers & 4 & 1,7 \\
Undetermined & 22 & 9,4 \\
Total & 232 & 100 \\
\hline
\end{tabular}

ACEIs : Angiotensin-converting enzyme inhibitors

\section{Discussion}

The work we present reflects hospital prevalence, prevalence probably undervalued in light of selection biases: patients met the clinical selection criteria without performing brain CT scan, the temporary closure of the service.

Despite these difficulties, strokes are a common reason for hospitalization: $32.4 \%$ in neurology and in our series, they represent more than a quarter of our hospitalizations.

The median age was 58.2 years and one quarter of strokes occur in people under 50. The occurrence of strokes at a relatively young age in developing countries is due, at least in part, to the significant percentage of some risk factors (smoking alcoholic); the relatively young general population, including the management of risk factors, education and public awareness on the concept of care for chronic diseases such as hypertension, diabetes mellitus and cardiovascular diseases. In this population about 6 out of 10 patients $(60 \%)$ had low socioeconomic status reflecting the difficulties in the daily management and realization of explorations. Jaillard et al [2] also recognize that low income, associated with illiteracy and unemployment significantly influenced the prognosis of stroke in general.

In Africa, more than $90 \%$ of patients with hemorrhagic stroke and more than half with ischemic stroke are found to have high blood pressure [3]. Hypertension remains the most important modifiable cardiovascular risk factor of ischemia and hemorrhage in both sexes and regardless of age $(79.3 \%$ in our series). This is usually an ignored or poorly controlled hypertension, found by other African studies [4, 5, 6, and 7]. In a study in South Africa, 58\% patients had never had their blood pressure measured. This does not necessarily mean that high blood pressure is not a problem, but rather that hypertension as a risk factor for disease in this population does not receive much attention [8]

It is imperative to conduct a health policy focused on primary, collective and individual prevention.
Alcoholism is found in our series with $27.1 \%$ of patients; it is the second risk factor in Gabon according to Kouna [9]. Tobacco is the third risk factor in our work; this could be explained by the importance of smoking in urban areas and among the younger population. In the series of Kouna [9], smoking represents $16.2 \%$ of cases. For Duc et al. [10], smoking triples the risk of ischemic stroke by an arterial vasoconstriction mechanism and platelet aggregation. We found a significant percentage of recurrent strokes (21.6\%).

Many factors could explain the occurrence of this recurrence: Patient Information problem on the disease, its risk factors, but also the care of patient. We emphasize the need to develop own training strategies to our realities: training of community health center doctors often in the first line in the care of patients and popularize the international recommendations measures in the context of secondary prevention.

In developed countries, the awareness of the early symptoms of stroke, telemedicine and the creation of stroke units have revolutionized the treatment of stroke in the acute phase.

In our series, the hospitalization period ranged from 0 to 60 days, irrespective of the type of stroke with an average of 6. 17 days

Only $19.8 \%$ of cases were admitted in our service within 24 hours. This could be explained by the lack of policy of population awareness but also, of the first-level medical personnel in the health pyramid of our country. This relatively long delay at admission contributed to postpone the early management, decisive element of the care.

Furthermore over half of patients $(52.2 \%)$ were admitted with a Glasgow score less than 11 which can be a predictive value of a guarded prognosis.

The presence of hemiplegia in almost $60 \%$ of patients, aphasia in one third of patients in a service with a single, not a speech therapist, physiotherapist poses real functional management difficulties, retards the start-up of adequate rehabilitation measures.

In the first 24 hours, only $5 \%$ of patients had access to CT; this percentage reflects both the ignorance of the signs of stroke and the long circuit and difficulties of access to imaging studies. MRI, available in some private and public structures, is rarely performed due to its average cost price of 125 US dollars (against 35 US dollars for CT) and the low rate of prescription because of the obvious diagnosis on $\mathrm{CT}$. In our series, thanks to the contribution of $\mathrm{CT}$, preponderance of ischemia is confirmed (59\%) and a high proportion of hematoma is noteworthy.

According to studies and countries, the predominance of stroke type varies $[11,6,12,7]$. But, most of these studies report a slightly higher percentage of ischemia compared to hemorrhage as reported in this work. This high percentage of cerebral hematoma in various African studies could be explained in most cases by poor adherence in the treatment of hypertension or, sometimes, an ignorance of this chronic disorder responsible for $74 \%$ of $\mathrm{ICH}$ (intracerebral 
hemorrhage) in our series.

Apart from conventional explorations (biological and cardiac), we had to realize in about $10 \%$ of AIS, a lumbar puncture in the etiologic research of infectious vasculitis.

HIV prevalence in our regions is about $5 \%$. In the study of Kouna in Gabon 15.5\% of patients tested were infected with HIV [9] In South Africa, among the young people not addicts, $16 \%$ of young patients of stroke occurred in patients infected with HIV [13]. In our work environment research of infectious vasculitis by the achievement of the PL and testing for HIV should be systematic.

Vascular causes (atherosclerosis, vasculitis and other vascular causes) were found in $47 \%$ of patients with ischemia Specific etiologies of vasculitis have not always been found. More expensive examinations were necessary. But, given the modest socio-economic level, lots of patients have not been able to continue forward investigations. This was probably the same for heart disease, which accounted for $15 \%$ of the causes of AIS, rate underestimated given the insufficiency of cardiac imaging realization. The inadequacy of the technical equipment, the difficulties of explorations, and the rate of AIS without classically described causes in the literature partly explain the $37 \%$ of undetermined causes.

Hypertension was the main cause of ICH. It was apparent from our study that $74 \%$ of ICH were hypertensive origin .There are two types of lesions involved according to Cambier J. et al. [14]: Charcot-Bouchard aneurysms and arteriolar lipohyalinosis associated with chronic hypertension. The finding was the same with $15 \%$ undetermined causes.

Therapeutically, in secondary prevention, focus is on the correction and treatment of cardiovascular risk factors especially hypertension which remains worrying. Thus, the combination of angiotensin-converting enzyme inhibitors (ACEIs) and diuretics was the most prescribed therapy because of our patients' profile.

In this work, we found that one in four patients hospitalized for stroke dies from complications due to stroke. This finding may be explained by delayed diagnosis and management of stroke in our context. This rate is much lower than that reported by Dewar et al. [12] in South Africa and that of Kwasa al. [15] in Kenya which respectively recorded $38 \%$ and $46 \%$ of deaths in their samples.

However, decubitus complications were a concern, affecting $37 \%$ of the subjects in our workforce with an emphasis given to bedsores (50\%). Adequate preventive measures: adopt anti bedsore mattresses, achieve proper nursing, initiate early a physiotherapy are all simple and urgent measures to practice every day but sometimes difficult to achieve in our practice.

There are several important messages in this paper. The mean age of the patients was 58 years, about

10-15 years younger than patients with stroke in developed countries. The study provides no data on stroke subtype, but the high early case fatality could be due to a high proportion of hemorrhagic stroke. There are few data available on stroke subtype in sub- Saharan Africa, although hemorrhagic stroke may be more prevalent than in developed countries. [16]

Data suggest that stroke is already a major health problem in African countries, health-care systems are already overstretched, the burden of stroke will increase in the future, and cheap interventions are available.

Long-term follow-up of stroke patients is extremely difficult because of the lack of central identification registries, few census reports, no address. The choice is therefore to accept hospital data or have no data at all.

Stroke units could be of benefit in Africa but primary prevention can reduce stroke occurrence and can be implemented for example: national strategies to reduce tobacco smoking, promote healthy lifestyles, and

Strokes and cardiovascular diseases will represent the main challenge of developing countries health care management systems in the coming years. [17]

\section{Conclusion}

In sub-saharian Africa countries strokes represent the first cause of disability in neurological departments. In Cote d'Ivoire, strokes have high hospital prevalence, with a very high socio-economic impact in terms of mortality and morbidity in the populations of young adults. Primary prevention of stroke remains a major challenge in our African countries. The management of this disease requires the opening of neurovascular units. Around these key structures, radiology accessible at all times, an organization of stroke sector, implementing a multidisciplinary collaboration: neurologist - radiologist - emergency physician - cardiologist - rehabilitation doctors.

\section{References}

[1] Aka- Anghui Diarra E, Kourouma MT, TANOH C. Distribution of neurological diseases: Experience of neurology department of University Hospital of Cocody. Journal of Neurology Neurosurgery Psychiatry 2015; 1: 16-24

[2] Jaillard A, Besson G, Hommel M. Prevention of ischemic stroke. The review of the practitioner $1998 ; 10 ; 3176-81$.

[3] Mensah G A. Epidemiology of stroke and high blood pressure in Africa. Heart 2008; 94: 697-705

[4] Garbusinski JM, Van Der Sande MA, Bartholome EJ et al. Presentation and outcome in developing countries: a prospective study in the Gambia. Stroke 2005; 36: 1388-1393.

[5] Lemogoum D, Degaute JP, Bovet P. stroke prevention, treatment, and rehabilitation in sub Saharan Africa. Am J Prev Med 2005; 95-101.

[6] Sagui F, M'Baye PS, Dubecq C et al. Ischemic and hemorrhagic stroke in Dakar, Senegal: a hospital based study. Stroke 2005; 36: 1844-1847.

[7] Sene Diouf F, Basse AM, Touré K, et al. prognosis of stroke in department of neurology of Dakar. Dakar Med 2006; 51: 17-21.

[8] Bonita R, Truelsen $\mathrm{T}$ : Stroke in sub-Saharan Africa: a neglected chronic disease 
[9] The lancet Neurology Vol 2 October 2003

[10] Kouna NP, Millogo A, Siéméfo Kamgang FP, Assengon-Zeh Y Epidemiological features and outcome of stroke at Libreville hospital (GABON) Ajns 2007 vol.26,N2

[11] Duc M, Jacquier A. cerebral circulatory insufficiency. Encycl Méd. Chir, CP Neurologie, 4-4-09 1345-1363.

[12] Ogun SA, Ojini FI, Ogungbo B et al. stroke in south west Nigeria: a 10 years review. Stroke 2005; 36: 1120-1122.

[13] Dewar SR. Stroke outcome in the absence of a structure rehabilitation program. Sem Afri Med J 1990; 78: 200-202.

[14] Hoffmann M, Berger JR, Nath A, Rayens M. Cerebrovascular disease in young, HIV infected, black Africans in the Kwazulu natal province of South Africa. J Neurovirol 2000; 6: 229-236
[15] Cambier J, Masson M, Dehen H. abrégé de Neurologie. Masson ; 1989, 6eme édition : 367-415.

[16] Kwasa Too, Lore W. stroke at kenyatta national hospital. East Afr Med J 1990; 67: 482-486.

[17] Imam I. Stroke: a review with an African perspective. Ann Trop Med Parasitol 2002; 96: 435-45.

[18] Balogou AAK, Belo M, Apetse K, Kombate D, Amouzouvi KD, Grunitzky EK. Stroke in patients 15 to 45 years in the neurology department of the University Hospital Lomé campus. Journal home 2006; vol 8, n2. 